\title{
L'HOMME L'Homme
}

164 | octobre-décembre 2002

Histoire, littérature et ethnologie

\section{David D. Gilmore, Misogyny: The Male Malady}

Philadelphia, University of Pennsylvania Press, 2001, XIII + 253 p., gloss., réf., index

\section{Pascale Bonnemère}

\section{OpenEdition}

Journals

Édition électronique

URL : https://journals.openedition.org//homme/14292

DOI : 10.4000//homme.14292

ISSN : 1953-8103

Éditeur

Éditions de l'EHESS

Édition imprimée

Date de publication : 1 décembre 2002

Pagination : 200-202

ISBN : 2-7132-1775-X

ISSN : 0439-4216

Référence électronique

Pascale Bonnemère, «David D. Gilmore, Misogyny: The Male Malady ", L'Homme [En ligne], 164 | octobre-décembre 2002, mis en ligne le 25 mars 2008, consulté le 24 avril 2022. URL : http:// journals.openedition.org//homme/14292 ; DOI : https://doi.org/10.4000//homme.14292

Ce document a été généré automatiquement le 24 avril 2022

(c) École des hautes études en sciences sociales 


\section{David D. Gilmore, Misogyny: The Male Malady}

Philadelphia, University of Pennsylvania Press, 2001, XIII + 253 p., gloss., réf., index

\section{Pascale Bonnemère}

ETHNOLOGUE spécialiste de l'Espagne, David Gilmore s'intéresse aussi depuis longtemps à des questions générales relevant du symbolisme du genre, de la sexualité et de la masculinité, comme l'atteste le titre d'un de ses ouvrages, Manhood in the Making: Cultural Concepts of Masculinity, paru en 1990. En un sens, le présent livre poursuit cette étude anthropologique de la masculinité puisqu'il cherche à interpréter l'universalité de l'attitude des hommes envers les femmes, faite de dénigrement, de rejet, de crainte, et par-dessus tout d'ambivalence. Son propos est double: mettre au jour les fondements de cette attitude qui, dans ses grandes lignes, transcende une société ou une époque particulière, et déterminer les facteurs qui pourraient expliquer les variations rencontrées et qui sont susceptibles d'amplifier ou, au contraire, d'atténuer les manifestations de cette « misogynie».

Bien que l'auteur assigne à ce terme un champ sémantique très large, son étude s'en tient aux dimensions symboliques de ce phénomène. Il n'est en effet nullement question de s'attarder sur la réalité pratique, quotidienne, du comportement des hommes vis-à-vis des femmes. Il s'agit plutôt de comprendre les représentations de la féminité et les implications rituelles de ces représentations. Dans l'introduction, Gilmore pose ainsi une série de questions : pourquoi les hommes, en tous lieux et en tous temps, craignent-ils et raillent-ils les femmes qui, après tout, au moins physiquement, sont le sexe faible? Quel danger les femmes représentent-elles pour eux ? Pourquoi les hommes se font-ils souffrir eux-mêmes autant qu'ils font souffrir les femmes dans leurs efforts pour échapper aux pouvoirs supposés néfastes de celles-ci? Pourquoi la pire misogynie semble-t-elle aller de pair avec une glorification de la féminité, avec des simulations de la menstruation, avec la couvade? (p. 11). Voilà le problème posé. 
3 David Gilmore s'attelle à la tâche avec érudition. Fondée à la fois sur des données ethnographiques, des livres traitant des grandes religions, des ouvrages de fiction ou des textes philosophiques, son analyse offre un large spectre. N'était la rigueur de la démonstration, l'abondance d'informations peut parfois rebuter le lecteur lorsque les mêmes données sont reprises pour illustrer les différents aspects de cet ensemble de représentations sur les femmes à travers les cultures et les époques; et ce n'est qu'une fois le livre refermé que la structure de l'ouvrage lui devient réellement intelligible.

4 Centrales dans ce livre sont les références à la Nouvelle-Guinée, dont les sociétés représentent pour l'auteur la misogynie poussée en quelque sorte à l'extrême. Dans cette région, l'accent est mis sur le danger associé au corps des femmes, notamment à leur vagin d'où s'écoule le sang menstruel, parmi d'autres fluides polluants. La sexualité elle-même est considérée comme une activité périlleuse, car pratiquée sans retenue elle épuise le stock de sperme que les hommes ont en quantité limitée. La NouvelleGuinée, comme l'Amazonie à laquelle David Gilmore se réfère également, représente ce qu'il appelle la «body-oriented misogyny» (p. 136). Mais il existe un autre type de misogynie, qualifié de «spiritual-intellectual» (p.137), qui affuble les femmes de traits de caractère malfaisants : elles sont alors sournoises, empoisonneuses ou commères et se présentent sous la forme de sorcières, de marâtres ou de viragos. L'auteur trouve des exemples de tels êtres absolument indignes de confiance dans des écrits aussi différents que les mythes gréco-romains, les textes hindous ou les contes de Grimm.

$5 \quad$ L'idée qui sous-tend ce vaste tour d'horizon au sein des cultures et des religions passées et présentes est évidemment de montrer que partout dans le monde et à toutes les époques les représentations attachées aux femmes revêtent des formes similaires. L'ambivalence est sans doute le terme qui caractérise le mieux les rapports que les hommes entretiennent universellement avec la féminité. Car, au dénigrement généralisé des femmes s'ajoute souvent ce que Gilmore qualifie, assez maladroitement, de "gynophilie » (chap. X). Il désigne par ce terme aussi bien des pratiques rituelles comme le "penis- [ou nose-] bleeding» (en Mélanésie) ou la couvade (en Amazonie), que les saignées opérées pour des raisons médicales au cours des siècles passés, ou les récits du folklore européen mettant en scène des hommes enceints. La plupart des spécialistes ont interprété ces pratiques en faisant référence aux fonctions physiologiques féminines, que les hommes chercheraient à imiter. La menstruation est ainsi considérée comme étant à l'origine d'une meilleure santé des femmes, car l'écoulement régulier de sang permet d'éliminer les impuretés accumulées dans le corps. Quant à la couvade, qui consiste en ce que le futur père observe les mêmes interdits que sa femme enceinte, restreint ses mouvements et imite les douleurs de l'enfantement, elle viserait à rendre la relation d'un père à sa progéniture équivalente à celle qui unit la mère à ses enfants (p. 191).

6 Pour rendre compte de cette situation ambivalente où les femmes sont dénigrées mais où leurs fonctions procréatrices et nourricières font l'objet d'une imitation rituelle par les hommes, et ce de manière universelle ou quasi universelle, il faut recourir, soutient Gilmore, à des explications de type psychologique qui postulent que tous les êtres humains - ceux du même sexe en tout cas - sont sujets au cours de leur développement aux mêmes situations et aux mêmes conflits psychiques.

7 Plusieurs théories ont été proposées pour rendre compte de l'universalité de la misogynie. La plupart se fondent sur les interprétations freudiennes de la psychologie masculine et postulent que le lien du garçon avec sa mère détermine les relations qu'il 
entretiendra avec les femmes dans sa vie d'adulte (p.156). Parce qu'il se sent totalement dépendant d'elle, le petit enfant développe des sentiments empreints d'ambivalence envers cette figure maternelle omnipotente, passant tour à tour de l'amour à la haine et au ressentiment (p.157). Certains auteurs pensent que cette relation entraîne une identification à la mère qui est ensuite vécue par le garçon comme un obstacle à sa masculinité. Le processus de désidentification par lequel le garçon doit alors passer prend la forme d'un rejet absolu de tout ce qui est associé à celle-ci, rejet qui serait à la source du dénigrement masculin à l'égard des femmes. Notons au passage que l'initiation masculine a souvent été interprétée comme un rituel visant à opérer cette mise à distance du garçon et de sa mère.

Si la psychologie permet ainsi d'expliquer les caractères universels de la misogynie, elle n'est pas d'un grand secours pour rendre compte des variations rencontrées dans l'expression de cette attitude. Seuls les facteurs sociaux sont en effet à même de le faire. Gilmore rappelle que, selon les premiers ethnologues de la Nouvelle-Guinée, le degré d'antagonisme sexuel varie avec les formes du mariage: il serait plus élevé lorsque les épouses proviennent de groupes ennemis. Une idéologie fortement patrilinéaire, qui souligne la solidarité entre les hommes liés par la consanguinité, favoriserait également l'idée que les femmes sont des étrangères auxquelles on ne peut guère accorder sa confiance. Des critiques et des aménagements ponctuels de l'hypothèse de Mervyn $\mathrm{J}$. Meggitt ${ }^{1}$ ont été proposés ici et là - notamment par Marilyn Strathern ${ }^{2}$ dans son livre de 1972, qui ne figure pourtant pas dans la bibliographie -, mais sans que celle-ci soit réellement débattue. Des travaux comparatifs récents, menés par exemple dans les groupes anga - dont les Baruya et les Sambia, cités par Gilmore, sont les plus fameux représentants - suggèrent l'existence de correspondances fortes entre la manière dont les actes rituels des initiations masculines sont préparés et mis en scène, leur contenu et les représentations des processus physiologiques de la reproduction. À son tour, cette configuration symbolico-rituelle rejaillirait sur la façon de considérer les femmes ${ }^{3}$. Or, les résultats de ces travaux de micro-comparatisme confirment bien plus les intuitions clairvoyantes de Kenneth E. Read ${ }^{4}$ que les propositions sans nuances de l'étude plus largement comparative menée par Meggitt, douze ans plus tard.

9 Sans doute parce que celle-ci relève de l'ordre politique et social, alors que Read ancre aussi ses analyses dans la dimension psychologique du réel, Gilmore juge les premiers plus adéquats pour illustrer le propos de ses chapitres V ("Social Structure») et IX («Structural and Materialist Theories»). Pourtant, force est d'admettre que, malgré la mise au jour de telles corrélations ponctuelles entre le degré de misogynie et l'organisation sociale, cette conduite masculine est de facto compatible avec des formes d'organisation économique, religieuse, politique et sociale extrêmement variées.

C'est pourquoi seule la psychologie peut, selon l'auteur, expliquer «la nature récurrente des croyances magiques, des illusions et des fantasmes que la misogynie transporte avec elle » (p. 220). Pour autant, si l'origine de celle-ci est à chercher dans le psychisme masculin, les expressions et l'intensité particulières qu'elle revêt dans telle ou telle situation ethnographique ou historique ne peuvent être comprises que grâce à la prise en compte de facteurs externes, qu'ils soient environnementaux, économiques ou sociaux. À l'issue de son livre, l'auteur dévoile sa perspective qu'il qualifie lui-même d'éclectique en ce qu'elle combine plusieurs théories psychologiques évoquées dans les chapitres précédents. Pour lui, seule une telle combinaison permet d'expliquer la 
misogynie dans toute sa richesse et sa diversité (p. 227). On ne peut que souscrire à cette analyse qui mêle les interprétations psychologiques, seules à même de rendre compte d'un phénomène aussi universel et les explications culturelles et sociologiques, seules capables de donner un sens aux variations, si minimes soient-elles, rencontrées à des époques et en des lieux particuliers. La route est encore longue pour comprendre les fondements de ces variations, mais la publication récente d'un ouvrage consacré aux formes du gender en Amazonie et en Mélanésie ${ }^{5}$ montre que le comparatisme mené à des échelles variées, dont celle, indispensable, de la micro-comparaison, a encore de beaux jours devant lui.

\section{NOTES}

1. Mervyn J. Meggitt, « Male-female relationships in the Highlands of Australian New Guinea ", American Anthropologist, 1964, 66 : (Numéro special : A New Guinea. The Central Highlands, James B. Watson, ed.) : 204-224.

2. Marilyn Strathern, Women in Between : Female Roles in a Male World, Mount Hagen, New Guinea, London, Seminar Press, 1972.

3. Cf. Pascale Bonnemère, Le Pandanus rouge. Corps, différence des sexes et parenté chez les Ankave-Anga de Papouasie Nouvelle-Guinée, Paris, CNRS Éditions/Éditions de la Maison des sciences de l'homme, 1996 : chap. X.

4. Kenneth E. Read, « Nama cult of the central Highlands, New Guinea », Oceania, 1952, $23: 1-25$.

5. Cf. Thomas Gregor \& Donald Tuzin, eds, Gender in Amazonia and Melanesia. An Exploration of the Comparative Method, Berkeley, University of California Press, 2001.

\section{AUTEUR}

\section{PASCALE BONNEMÈRE}

CNRS, Centre de recherche et de documentation sur l'Océanie, Université de Provence, Aix-en-Provence. 